\title{
Chikungunya Epidemic in the French Overseas Territories using Syndromic Surveillance
}

\author{
Anne Fouillet ${ }^{\star 1}$, Jacques Rosine ${ }^{2}$, Vanina Bousquet ${ }^{1}$, Sylvie Cassadou ${ }^{3}$, Luisiane \\ Carvalho $^{4}$, Martine Ledrans ${ }^{2}$, Audrey Andrieu ${ }^{2}$, Thierry Cardoso ${ }^{1}$ and Céline Caserio- \\ Schönemann ${ }^{1}$
}

${ }^{1}$ French Institute for Public Health Surveillance, Saint Maurice, France; ${ }^{2}$ French Institute for Public Health Surveillance, Fort-deFrance, Martinique; ${ }^{3}$ French institute for Public Health Surveillance, Pointe-à-Pitre, Guadeloupe; ${ }^{4}$ French Institute for Public Health Surveillance, Cayenne, French Guiana

\section{Objective}

Description of the temporal pattern of the chikungunya epidemic and the characteristics of patients in the French overseas territories of Americas using the French syndromic surveillance system SurSaUD.

\section{Introduction}

In December 2013, an emergence of chikungunya was observed in the French Caribbean region. Starting on the Saint-Martin island, the epidemic of chikungunya spread in Martinique on December 2013. The first cases were then observed in Guadeloupe in December 2013 and in January 2014 in the French Guyana [1].

A specific surveillance system has been implemented based on a sentinel general practitioners' network enabling the estimation of the number of cases clinically suggestive [2]. Severity of this arbovirus is assessed using the number of hospitalized cases.

The syndromic surveillance system SurSaUD, based on the daily collection of two complementary morbidity data sources, is also implemented in these territories and has contributed to the surveillance of this outbreak.

\section{Methods}

Individual data collected in two emergency departments (ED) in Guadeloupe and one ED in French Guyana are daily transmitted and analyzed by the French institute for public health surveillance (InVS). In Martinique, the emergency departments are not still included in the syndromic surveillance network but data from the emergency general practitioners' association SOS Médecins are daily collected and analysed by InVS.

In both data sources, individual demographic (age, gender, place of residence), administrative (date of visit) and medical information (chief complaints, medical diagnosis, hospitalization after the consultation) are available.

The weekly number of ED attendances with the medical diagnosis coded "A92.0" (ICD10) in Guadeloupe and French Guyana and the weekly number of consultations in the GP's association SOS Médecins with a clinical diagnosis of chikungunya in Martinique have been compared with the dynamic of the estimated number of cases clinically suggestive provided by the specific surveillance system. The outbreak has also been described by age group and gender.

\section{Results}

In Guadeloupe and in French Guyana, the temporal evolution of the number of attendances with a chikungunya diagnosis is correlated with the evolution of the epidemic followed by the specific surveillance system. In Guadeloupe, patients who visited the ED are mainly children aged less than 15 . This diagnosis is the third pathology recorded in the ED for this age group from January to August 2014 (after trauma and ENT infection). The distribution of patients by age group is different between the two ED.
Because of the location of the GP's association SOS Médecins in a part of Martinique, the pattern of the number of consultations is highly correlated with the outbreak followed with the specific surveillance system during the first step of the outbreak but is less correlated when the epidemic spread into the other parts of the island. In this data source, the patients with a chikungunya diagnosis are older (50 years old in average) than patients with another diagnosis (44 years old).

\section{Conclusions}

The specific and syndromic surveillance systems ensure a complementary surveillance of chikungunya outbreak. The added values of syndromic surveillance data sources are the reactivity (daily collection) and the availability of individual information enabling description of patients and identification of the most vulnerable population groups. The specific surveillance system based on sentinel GP's network allows a representative surveillance of cases in the population, enabling an estimation of the number of cases in the population.

\section{Keywords}

Chikungunya; Caribbean area; France; Emergency department; GP's association

\section{Acknowledgments}

The authors thank the emergency departments and the association SOS Médecins for providing data and their contribution to the surveillance.

\section{References}

[1] Van Bortel W, Dorleans F, Rosine J, et al. Chikungunya outbreak in the Caribbean region, December 2013 to March 2014, and the significance for Europe. Euro Surveill.2014;19(13):pii=20759.

[2] Ledrans M, Cassadou S, Boucau S, et al. Émergence du chikungunya dans les départements français d'Amérique : organisation et résultats de la surveillance épidémiologique, avril 2014. Bull Epidémiol Hebd. 2014 (21-22):368-79.

*Anne Fouillet

E-mail: a.fouillet@invs.sante.fr 